\title{
POSITRON BEAM PROPAGATION IN A METER LONG PLASMA CHANNEL
}

\author{
K. A. Marsh, B. E. Blue, C. E. Clayton, C. Joshi, W. B. Mori, UCLA, Los Angeles, CA, F.-J. \\ Decker, M. J. Hogan, R. Iverson, C. O'Connell, P. Raimondi, R. Siemann, D. Walz, SLAC, Stanford, \\ CA, T. C. Katsouleas, P. Muggli, USC, Los Angeles, CA
}

\begin{abstract}
Recent experiments and simulations have shown that positron beams propagating in plasmas can be focused and also create wakes with large accelerating gradients. For similar parameters, the wakes driven by positron beams are somewhat smaller compared to the case of an electron beam. Simulations have shown that the wake amplitude can be increased if the positron beam is propagated in a hollow plasma channel (Ref. 1). This paper, compares experimentally, the propagation and beam dynamics of a positron beam in a meter scale homogeneous plasma, to a positron beam hollow channel plasma. The results show that positron beams in hollow channels are less prone to distortions and deflections. Hollow channels were observed to guide the positron beam onto the channel axis. Beam energy loss was also observed implying the formation of a large wake amplitude. The experiments were carried out as part of the E-162 plasma wakefield experiments at SLAC.
\end{abstract}

\section{INTRODUCTION}

In the "blow out" regime of the plasma wakefield accelerator (PWFA) a high density electron beam exerts a large radial force on the plasma electrons, creating a nearly uniform ion column. As the beam passes through, plasma electrons rush back to neutralize the ions creating a large amplitude wake following the drive beam. The wake can be used to accelerate electrons in a trailing beam, or electrons in the tail of the drive beam can be used to probe the wake.

The ion column acts as a thick, underdense, plasma lens, with a large focusing strength thus producing multiple betatron oscillations as shown in reference 2 . For a uniform ion column the force on the beam is linear with radius. In this case, the beam can propagate without distortion or emittance growth.

In the case of a positron beam, plasma electrons flow in to neutralize the beam in a very dynamic way. As the beam passes through, these plasma electrons rapidly exit leaving a large amplitude wake behind. Due to the large reservoir of plasma electrons surrounding the beam, electrons can be pulled in continuously. Some overshoot and pass through the beam or oscillate about the beam axis. The resulting focusing forces are nonlinear and vary with $\mathrm{r}$ and $\mathrm{z}$ along the beam as shown in Ref. 3. At best, nonlinear but symmetric forces, result in emittance growth and beam halo formation.

A radially inhomogeneous plasma can create a deflection or distortion of an electron beam due to the creation of an asymmetric ion column. Similarly, positron beams can be deflected or distorted due to a radially asymmetric flow of plasma electrons to one part of the positron beam or another. Also, electron or positron beams with head to tail tilts can be deflected and distorted. This is because forces on axially misaligned beam slices will have a focusing component plus a transverse deflecting component. These distortions and deflections are experimentally unwanted and have often been observed during E-162 runs.

Hollow channel plasmas can guide a positron beam. Ions on the channel wall repel the beam and confine it on axis. The physical mechanism and results are discussed further below.

\section{EXPERIMENTAL SET UP}

The experiment was conducted at SLAC as part of the PWFA work known as E-162. The experiment was located at the Final Focus Test Beam Facility (FFTB). The set up and measurement details are described in many other papers (See references). Here we give only a brief description. The $28.5 \mathrm{GeV}$ positron beam was focused to the entrance of a uv photoionized lithium plasma. Typical beam parameters were, $2 \times 10^{10}$ positrons per bunch, $600 \mu \mathrm{m}$ rms bunch length, with a $25 \mu \mathrm{m}$ beam size at the plasma entrance. The SLAC beam emittance was increased due to scattering from foils and from a thin uv coupling mirror placed in the beam line. The beam emittance at the plasma entrance was about $2 \times 10^{-9} \mathrm{~m}-\mathrm{r}$.

A column of lithium vapor, $140 \mathrm{~cm}$, long was produced in a heat pipe oven (Ref. 4). A uv ArF laser was used to single photon ionize the lithium vapor. For these experiments, the plasma density was proportional to uv fluence $\left(\mathrm{J} / \mathrm{cm}^{2}\right)$. The plasma density ranged from $(0-$ 2) $\times 10^{14} \mathrm{~cm}^{-3}$. An axially uniform plasma can be produced by focusing the laser to compensate for uv absorption. Axial variations in plasma density do not significantly alter the resulting transverse dynamics of the beam as long as the scale of the density variation is larger than the betatron wavelength. When absorption is taken into account, the radial plasma profile mimics the laser profile. 
The plasma channel was produced by placing a mask in the center of the uv laser beam. The mask diameters used were $1 \mathrm{~mm}$ and $.6 \mathrm{~mm}$. An ideal plasma channel with rigid walls like that described in Ref. 1, would be difficult to produce due diffusion of the plasma. Since the laser fires $\sim 200 \mathrm{~ns}$ before the positron beam arrives, this allows some time for diffusion to take place. We believe the beam is propagating in something more like a density depression than an ideal rigid channel. In any case this will be referred to as a hollow channel as well. Comparison to the case without a channel was performed for the same conditions by removing the mask.

The beam spot size and location was monitored by optical transition radiators (OTR) located upstream (US) and downstream (DS) of the plasma. Focusing and transverse dynamics were observed on the DSOTR verses a uv count which is proportional to the plasma density. Monitoring of the incoming beam parameters was a simple matter of toggling the uv laser so that no plasma was produced.

An imaging spectrometer was set up to monitor the changes in beam energy after traversing the plasma. A permanent dipole bending magnet was used to disperse the beam and quadrupole lenses were used to image the beam from the plasma exit to an aerogel Cherenkov radiator (CR). Imaging assures that the observed beam energy is true and not due to transverse effects. The magnetic dispersion is about $10 \mathrm{~cm}$ or $300 \mathrm{MeV} / \mathrm{mm}$. The CR was imaged onto a CCD camera where changes of the beam energy were observed. CR light was also imaged on to a streak camera (SC) where changes in the beam energy along the pulse were monitored.

The incoming beam has a correlated energy spread of about $350 \mathrm{MeV}$ with the higher energy in the head. Observation of energy gain of tail electrons is difficult unless they gain enough energy to be clearly seen above the energy of the head. By streaking the beam energy in time, we can look at the change in energy along the beam, particularly the energy loss of the middle of the beam and energy gain of the tail electrons.

\section{RESULT S}

Figure 1 compares the beam images at the DSOTR with no plasma, to plasma with and without a channel. With the channel the beam propagated and focused without distortion. Without a channel the beam was focused, but has an unwanted distortion. Figure 2 shows the average beam deflection verses uv counts, without the hollow channel. All the points are for the same input beam parameters. The beam was deflected without the channel indicating the plasma profile was radially inhomogeneous and or the beam was tilted. In contrast the beam was not deflected but rather guided with the hollow channel. Guiding occurs when the positron beam is misaligned or at a small angle to the hollow channel axis. The build up of ions on the channel wall, confine the beam by repelling the beam toward the channel axis.

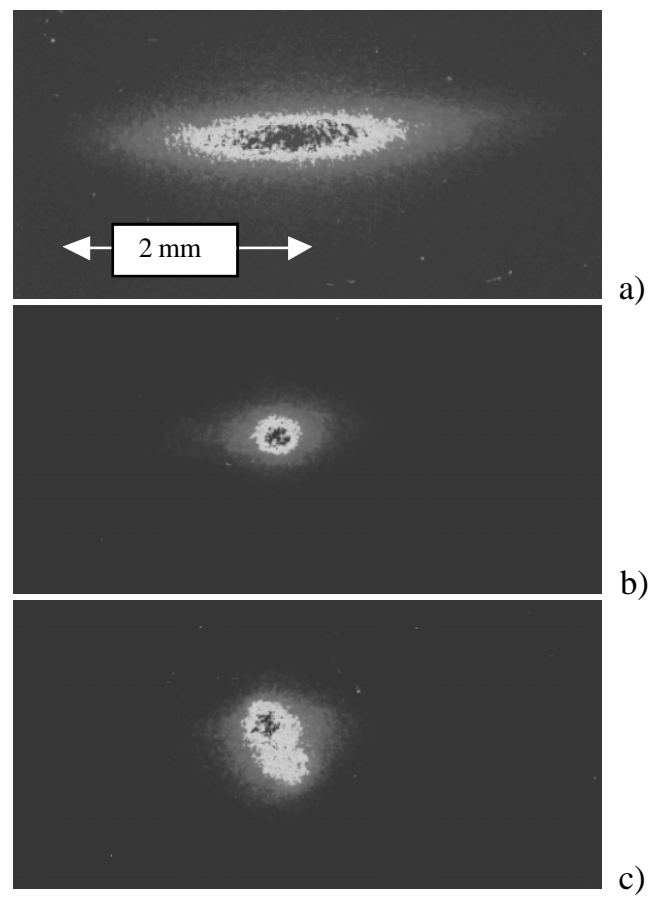

Figure 1. Comparison of positron beam images at the DSOTR for a) no plasma b) plasma with a hollow channel c) plasma without a channel .

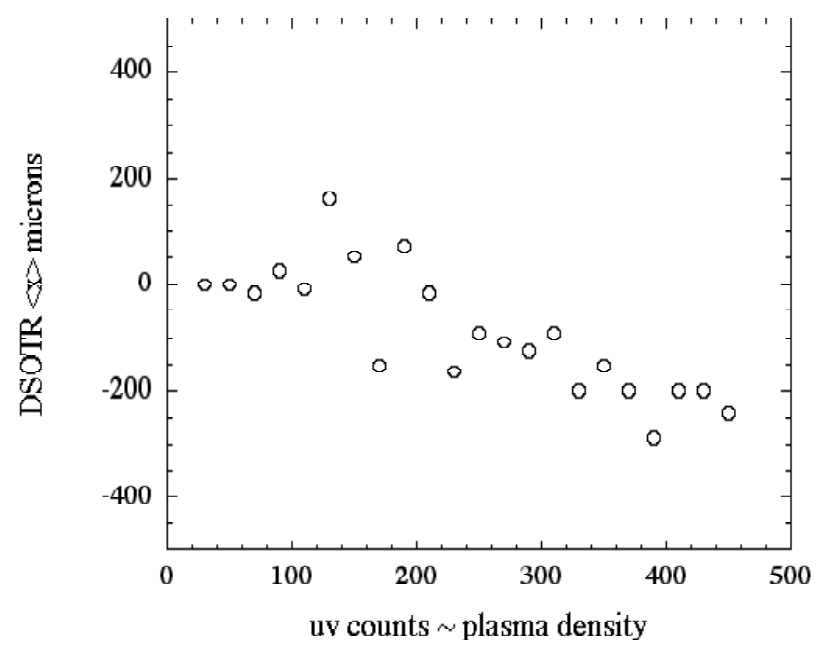

Figure 2. Average beam deflection verses uv counts (proportional to plasm density) for a plasma without a hollow channel.

Random shot to shot misaligment of the beam to the hollow channel axis occurred due to small energy variations of the beam. This resulted in steering from the slightly misaligned final focusing quads. Figure 3 shows the beam trajectory at the US and DS OTR's for 200 shots. The open circles show the correlation between beam location on the OTR's without plasma. The crosses 
show the beam guiding with the hollow channel. The beam moves toward the center location in $\mathrm{x}$, which demonstrates hollow channel guiding. Similarly the beam was also guided in $\mathrm{y}$.

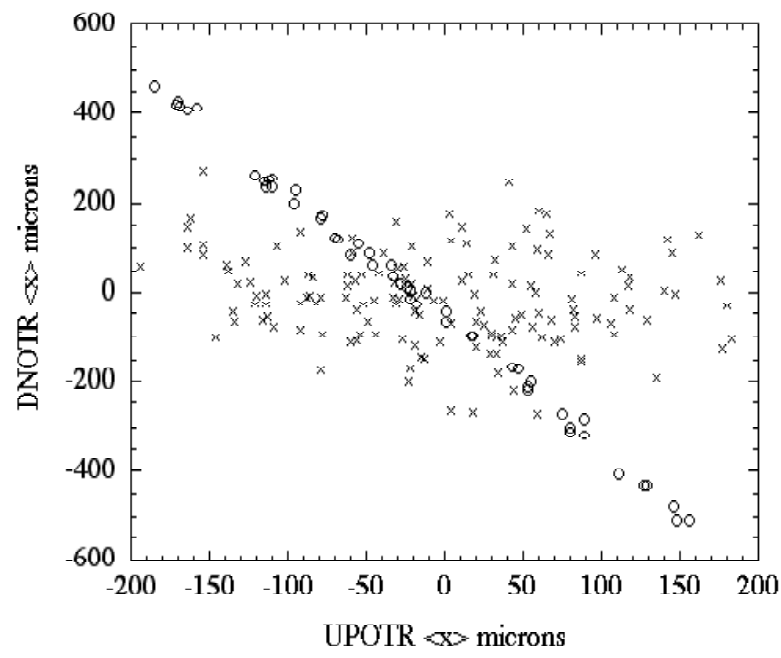

Figure 3. Beam guiding trajectory on DS vs. US OTR. The open circles show the correlation between beam location on the OTR's without plasma. Crosses show when the plasma is on the beam moves toward the center location of the channel in $\mathrm{x}$.

As noted above, the homogeneous plasma actually has a radial profile peaked on axis like the uv laser. Beams not on axis with the plasma were deflected. A beam on a trajectory moving across the plasma axis would appear to steer toward the axis. This is not guiding as evidenced by the fact that the beam is deflected further as the plasma density was increased.

The plasma dynamics of a positron beam propagating in a hollow channel are illustrated in Fig. 4a. An annulus of plasma electrons are pulled toward the beam as the beam moves through the channel. Most of the plasma electrons reach the beam axis as it passes by. Therefore very few plasma electrons cross the beam allowing for propagation with low distortion and deflection. Figure $4 \mathrm{~b}$, shows the flow in of plasma electrons for the homogeneous case. Plasma electrons cross the beam and some oscillate about the axis leading to nonlinear focusing forces along the beam.

The central portion of the beam looses energy as it propagates through the plasma channel. Figure 5, compares CR images of the energy spectra for no plasma, a low density hollow channel and a high density hollow channel plasma. At low density we see the beam is focused, but no significant energy change. At high density the beam looses significant energy of about 200 $\mathrm{MeV}$. To observe energy gain of the beam tail requires further analysis of the streak camera images.
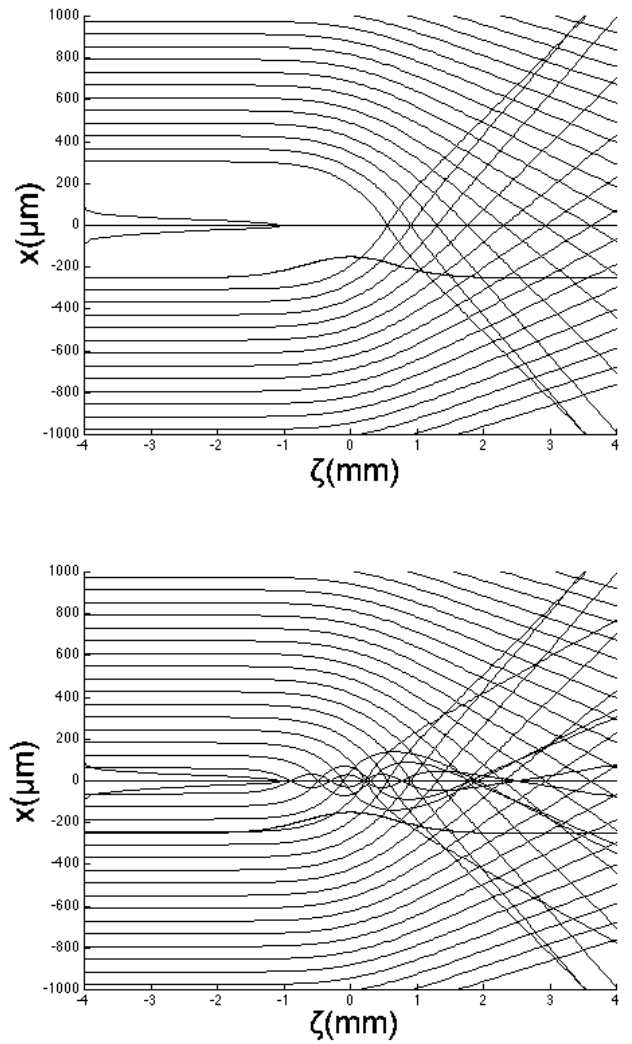

Figure 4. Illustration of plasma dynamics, a) with and b) without a hollow plasma channel. Beam moves to the left.

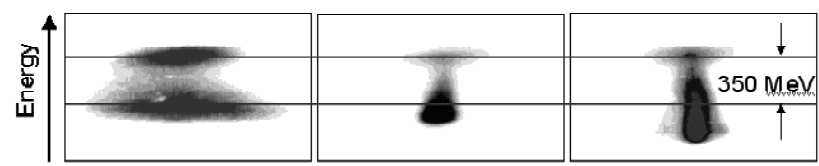

Figure 5. Energy spectrum of positron beam a) with no plasma compared to b) low density and c) high density hollow plasma channels.

\section{REFERENCES}

[1] S. Lee et al., Phys. Rev. E 64, 045501(R) (2001)

[2] C. E. Clayton et al., Phys. Rev. Lett. 88, 154801 (2002); C. O'Connell et al., PRST-AB 5, 121301 (2002)

[3] M. J. Hogan et al., Phys. Rev. Lett. accepted for publication; P. Muggli et al., these proceedings

[4] P. Muggli et al., IEEE Trans. Plasma Sci. 27, 791, (1999); K. A. Marsh et al., Advanced Accelerator Concepts, $10^{\text {th }}$ Workshop, Mandalay Beach, CA, Ed. C. E. Clayton, P. Muggli, AIP Conf. Proc. \#647, 2002, pp. 614-619.

We gratefully acknowledge our collaborators past and present, Brian "Photoshop" Flores and our DOE support. Work supported by DOE grants DE-FG03-92ER40727 andDE-FG03-92ER40745. 